\title{
374.
}

\section{ON THE HIGHER SINGULARITIES OF A PLANE CURVE.}

[From the Quarterly Journal of Pure and Applied Mathematics, vol. vII. (1866), pp. 212-223.]

THE theory of the singularities of a plane curve was first established by Plücker in his great work the Theorie der Algebraischen Curven, (1839), where he establishes, in regard to the ordinary singularities, a system of six equations; viz. if we have

$$
\begin{aligned}
& m \text {, the order of the curve, } \\
& n, \text {, class, } \\
& \delta, " \text { number of double points, } \\
& \kappa, ", \quad, \quad \text { cusps, } \\
& \tau, \quad, \quad, \quad \text { double-tangents } \\
& \kappa, ", \quad \text { inflexions, }
\end{aligned}
$$

then Pliicker's six equations are

$$
\begin{aligned}
& n=m(m-1)-2 \delta-3 \kappa \\
& \iota=3 m(m-2)-6 \delta-8 \kappa \\
& \tau=\frac{1}{2} m(m-2)\left(m^{2}-9\right)-\left(m^{2}-m-6\right)(2 \delta+3 \kappa)+2 \delta(\delta-1)+6 \delta \kappa+\frac{9}{2} \kappa(\kappa-1), \\
& m=n(n-1)-2 \tau-3 \iota \\
& \kappa=3 n(n-2)-6 \tau-\delta \iota \\
& \delta=\frac{1}{2} n(n-2)\left(n^{2}-9\right)-\left(n^{2}-n-6\right)(2 \tau+3 \iota)+2 \tau(\tau-1)+6 \tau \iota+\frac{9}{2} \iota(\iota-1),
\end{aligned}
$$

equivalent to three equations; thus $m$ and (within proper limits) $\delta$ and $\kappa$ may be considered arbitrary, and the first three equations then give $n, \iota, \tau$; and in like manner $n$ and (within proper limits) $\tau$ and $\iota$ may be considered as arbitrary, and the equations then give $m, \kappa, \delta$. 
I have used the ordinary expressions double points, cusps, double tangents, inflexions; but (using as I have elsewhere done ineunt as the correlative of tangent) it would be more precise and symmetrical to say, double ineunts, stationary ineunts, double tangents, and stationary tangents. The double ineunt is called also a node, viz. it is a crunode, or an acnode, according as the tangents are real or imaginary; and the stationary ineunt, or cusp, considered as (what in the theory of point-coordinates it in fact is) a particular case of the double ineunt, is a spinode; to render this notation symmetrical, we require certain new terms, say link, as the correlative to node, and flex as the correlative to cusp; then the double tangent is a link, viz. it is a colink, or an allink according as the ineunts upon it (points of contact) are real or imaginary; and the stationary tangent (inflexion) or flex, considered as (what in the theory of linecoordinates it in fact is) a particular case of the double tangent, is a relink. The ordinary singularities of a plane curve would thus be the node, the cusp, the link, and the flex; but $I$ shall retain the above-mentioned more usual expressions.

Deducible from the six equations, we have

$$
\begin{aligned}
n-m & =\frac{1}{3}(\iota-\kappa), \\
(n-m)(n+m-9) & =2(\tau-\delta),
\end{aligned}
$$

which are noticed by Plücker; and also the equation

$$
\frac{1}{2}(m-1)(m-2)-\delta-\kappa=\frac{1}{2}(n-1)(n-2)-\tau-\iota,
$$

recently noticed by $\mathrm{M}$. Clebsch, in connection with Riemann's investigations on the Abelian Integrals; a curve of the order $m$ may have $\frac{1}{2}(m-1)(m-2)$ double points, reckoning the cusp as a double point, and so a curve of the class $n$ may have $\frac{1}{2}(n-1)(n-2)$ double tangents, reckoning the inflexion as a double tangent; the two sides of this equation exhibit therefore, the right-hand side the deficiency of the actual from the possible number of double tangents, and the left-hand side the deficiency of the actual from the possible number of double points; and these two numbers are equal. We have a division into families based on the value of the expressions in question, or say on that of $\frac{1}{2}(m-1)(m-2)-\delta-\kappa$; when this is $=0$, that is, when the curve has its maximum number of double points (reckoning the cusp as a double point), the coordinates $x, y$ are expressible rationally in terms of a parameter $\theta$; when the number is $=1$, they can be expressed rationally in terms of $\theta$ and of the square root of a cubic or a quartic function of $\theta$, \&c. \&c. It thus appears that as well the number $\delta+\kappa$, as the combinations $2 \delta+3 \kappa$ and $6 \delta+8 \kappa$ which enter into Plücker's equations, plays an important part in the theory of the curve; the bearing of this remark will be seen in the sequel.

Plücker considers also some of the higher singularities; it will be convenient to mention two of his results.

No. 76, p. 216. If two branches of a curve touch each other, or more generally have a $g$-pointic intersection, the point in question is equivalent to $g$ double points, c. $\mathrm{v}$. 
and the tangent at this point to $g$ double tangents; hence, if there is no other point singularity, the equations give

$$
\begin{aligned}
n & =m(m-1)-2 g, \\
\iota & =3 m(m-2)-6 g, \\
\delta+g & =\frac{1}{2} m(m-2)\left(m^{2}-9\right)-\left(m^{2}-m-6\right) 2 g+2 g(g-1),
\end{aligned}
$$

the last of which may also be written

$$
\delta=\frac{1}{2} m(m-2)\left(m^{2}-9\right)-\left(m^{2}-m-g-4 \frac{1}{2}\right) 2 g .
$$

And Nos. 77-82, pp. 217-222. For a cusp of the second kind, we have

$$
\begin{aligned}
& n=m(m-1)-5 \\
& \iota=3 m(m-2)-15 \\
& \delta=\frac{1}{2} m(m-2)\left(m^{2}-9\right)-\left(m^{2}-m-7\right) 5
\end{aligned}
$$

these equations Plücker establishes by an independent algebraical investigation, and having done so, he remarks that they are deducible from the foregoing ones by writing therein $g=2 \frac{1}{2}$; that is, that the cusp of the second kind may be considered as equivalent to $2 \frac{1}{2}$ double points, and the tangent at the cusp to $2 \frac{1}{2}$ double tangents. And he thence passes to the cusp of a higher cusp equivalent to $h+\frac{1}{2}$ double points and $h+\frac{1}{2}$ double tangents. The results in this general case (although not, as in the original case, $g=2 \frac{1}{2}$, established independently) is perfectly correct; but the theory is open to a grave objection.

I remark, that assuming a certain singularity to be equivalent to the numbers $\delta^{\prime}$ of double points, $\kappa^{\prime}$ of cusps, $\tau^{\prime}$ of double tangents, and $\iota^{\prime}$ of inflexions, we have in the first instance to determine $\delta^{\prime}, \kappa^{\prime}, \tau^{\prime}$ and $\iota^{\prime}$ in such manner as to give in the class $n$, and in the numbers $\iota$ of inflexions and $\tau$ of double tangents, the reductions actually given by the singularity in question. Thus in the case of the cusp of the second kind, we ought to have

$$
\begin{aligned}
& 2 \delta^{\prime}+3 \kappa^{\prime}=5, \\
& 6 \delta^{\prime}+8 \kappa^{\prime}+\iota^{\prime}=15, \\
& \left(m^{2}-m-6\right)\left(2 \delta^{\prime}+3 \kappa^{\prime}\right)-2 \delta^{\prime}\left(\delta^{\prime}-1\right)-6 \delta^{\prime} \kappa^{\prime}-\frac{9}{2} \kappa^{\prime}\left(\kappa^{\prime}-1\right)+\tau^{\prime}=\left(m^{2}-m-7\right) 5,
\end{aligned}
$$

or, what is the same thing,

$$
2 \delta^{\prime}\left(\delta^{\prime}-1\right)+6 \delta^{\prime} \kappa^{\prime}+\frac{9}{2} \kappa^{\prime}\left(\kappa^{\prime}-1\right)-\tau^{\prime}=5 ;
$$

and so in general there are, for the determination of the four quantities $\delta^{\prime}, \kappa^{\prime}, \tau^{\prime}, \iota^{\prime}$, three equations. In the particular case these are satisfied by the values $\delta^{\prime}=2 \frac{1}{2}, \kappa^{\prime}=0$, $\tau^{\prime}=2 \frac{1}{2}, \quad \iota^{\prime}=0$, which are Pluicker's values; they are also satisfied by the values $\delta^{\prime}=1, \kappa^{\prime}=1, \quad \tau^{\prime}=1, \quad \iota^{\prime}=1$, which have the advantage of being integer instead of fractional.

But there is really a further condition to be satisfied, viz. the number $\delta^{\prime}+\kappa^{\prime}$ must have a certain definite value dependent on the nature of the singularity; for 
the case in hand, this is $\delta^{\prime}+\kappa^{\prime}=2$ (I obtain this by the consideration of a quartic curve, having a cusp of the second kind, and also a double point; $\delta+\kappa$ has here its maximum value $=3$; and as the double point gives $\delta=1$, the cusp of the second kind gives $\left.\delta^{\prime}+\kappa^{\prime}=2\right)$; and joining to the former conditions this new condition, we have definitely $\delta^{\prime}=1, \kappa^{\prime}=1, \tau^{\prime}=1, \iota^{\prime}=1$. I have elsewhere noticed, [343], that the cusp of the second kind was equivalent to a double point and cusp, and accordingly proposed to call it the node-cusp; but I had not then remarked that it was also necessary to treat the tangent as equivalent to a double tangent and a stationary tangent (or inflexion).

It appears from the foregoing considerations, that any singularity whatever is to be regarded as equivalent, and that in a perfectly definite manner, to a certain number $\delta^{\prime}$ of double points, $\kappa^{\prime}$ of cusps, $\tau^{\prime}$ of double tangents, and $\iota^{\prime}$ of inflexions; we have only to ascertain how for any given singularity the values of these numbers are to be ascertained; and when this is done, Pluicker's equations will be applicable to any singularities whatever of a plane curve.

At any point of a plane curve there is either one branch, or any number of branches, touching or not touching each other: taking the given point as origin, then for each branch the equation of the curve gives for the ordinate $y$ an expression of the form

$$
y=A x^{p}+B x^{q}+\ldots,
$$

where the series is arranged in ascending powers of $x$, and where the coefficients $A, B, \ldots$ have definite unique values; and, conversely, that which is given by such expression of $y$ is a branch of the curve. It is assumed that the axis of $y$, or line $x=0$, is not a tangent to the curve; this implies that the exponents $p, q, \ldots$ are none of them inferior to 1 , or, what is the same thing, that the lowest exponent $p$ is $=1$ at least: it is for the most part convenient to take the axis of $x$, or line $y=0$, a tangent to the branch; the lowest exponent $p$ is then $>1$.

The exponents may be all integer, and the branch is then said to be linear; or else the exponents or some of them may be fractional, and the branch is then superlinear; viz. in the latter case, assuming that the fractional exponents are all of them in their least terms, and that $\alpha$ is the least common multiple of the denominators (so that the expression for $y$ is a rational function of $x^{\frac{1}{a}}$ ), then the branch is quadric, cubic, \&c. according as we have $\alpha=2, \alpha=3$, \&c. It is clear that the expression for $y$ has precisely $\alpha$ values, viz. the values obtained by attributing to the radical $x^{\frac{1}{\alpha}}$ each of its $\alpha$ values. Corresponding to each of these $\alpha$ values, we have what I term a partial branch of the curve, so that the quadric branch is made up of two partial branches, the cubic branch of three partial branches and so on; for a linear branch or when $\alpha=1$, a partial branch is nothing else than the branch itself; and the expression a partial branch will accordingly include the case of a linear branch.

Suppose that at any point of the curve we have two partial branches, belonging or not belonging to the same branch; let these be referred to the same axes, the

$66-2$ 
axis of $y$ not being a tangent to either branch, so that the exponents are none of them $<1$. If in the series for $y_{1}-y_{2}$, (the difference of the two ordinates) the least exponent is $=P$, then (whether $P$ is integer or fractional) the two partial branches are said to have, at the given point, $P$ common points, or, more briefly, to intersect in $P$ points. We may from this definition calculate the number of intersections of two branches with each other, or of a branch with itself; for instance, suppose that at any point of the curve we have $(\alpha=6)$ the sextic branch

$$
y=x^{\frac{4}{3}}+x^{\frac{5}{2}}+\ldots,
$$

we have the six partial branches

$$
\begin{array}{ll}
y_{1}=x^{\frac{4}{3}}+x^{\frac{5}{2}}+\ldots, & y_{4}=x^{\frac{4}{3}}-x^{\frac{5}{2}}+\ldots, \\
y_{2}=\omega x^{\frac{4}{3}}+x^{\frac{5}{2}}+\ldots, & y_{5}=\omega x^{\frac{4}{3}}-x^{\frac{5}{2}}+\ldots, \\
y_{3}=\omega^{2} x^{\frac{4}{3}}+x^{\frac{5}{2}}+\ldots, & y_{6}=\omega^{2} x^{\frac{4}{3}}-x^{\frac{5}{2}}+\ldots ;
\end{array}
$$

hence calculating (what is most convenient) twice the number of intersections of the branch with itself, the partial branch $y_{1}$ intersects the other partial branches in $\frac{4}{3}, \frac{4}{3}, \frac{5}{2}, \frac{4}{3}, \frac{4}{3}$ points respectively, giving the sum $\frac{16}{3}+\frac{5}{2}=\frac{47}{6}$; each other partial branch intersects the remaining five branches in the same number of points; and therefore twice the number of intersections is $=47$.

For the singularity $y=x^{\frac{4}{3}}+x^{\frac{5}{2}}+\ldots$ in question, I say that if this be equivalent as above to $\delta^{\prime}$ double points, $\kappa^{\prime}$ cusps, $\tau^{\prime}$ double tangents and $\iota^{\prime}$ inflexions, then that the number 47 just obtained is the value of $2 \delta^{\prime}+3 \kappa^{\prime}$, and moreover, that the value of $\kappa^{\prime}$ is $\kappa^{\prime}=\alpha-1=5$; that is, we have $2 \delta^{\prime}+3 \kappa^{\prime}=47$ and $\kappa^{\prime}=5$; or, what is the same thing, $\delta^{\prime}=16 ; \kappa^{\prime}=5$. For the determination of the numbers $\tau^{\prime}, \iota^{\prime}$, it is to be observed that the foregoing theory of branches is a theory of the points of a branch, by means of point-coordinates: there is a precisely similar theory of the tangents of a branch by means of line-coordinates, and we may inquire as to the number of the common tangents of two partial branches; and thence as to the number of common tangents of two branches, or of a branch with itself-it will appear that the line-equation of the branch is $Z=X^{4} \ldots+X^{\frac{15}{2}} \ldots$, so that the branch (which is as to its points sextic, $\alpha=6)$ is as to its tangents quadric, $\beta=2$, the two partial branches have with each other the number $=\frac{15}{2}$ of common tangents, or twice this number is $=15$; that is, we have $2 \tau^{\prime}+3 \iota^{\prime}=15$, and moreover $\iota^{\prime}=\beta-1=1$, that is $\tau^{\prime}=6, \iota^{\prime}=1$; or finally for the singularity in question, the numbers $\delta^{\prime}, \kappa^{\prime}, \tau^{\prime}, \iota^{\prime}$ are $=16,5,6,1$ respectively.

And so generally in the case of a branch which is as to its points $\alpha$-ic, having with itself a number $=\frac{1}{2} M$ of common points; and as to its tangents $\beta$-ic, having with itself a number $=\frac{1}{2} N$ of common tangents, we have $2 \delta^{\prime}+3 \kappa^{\prime}=M, \kappa^{\prime}=\alpha-1$, $2 \tau^{\prime}+3 \iota^{\prime}=N, \iota^{\prime}=\beta-1$, or, what is the same thing, the values of $\delta^{\prime}, \kappa^{\prime}, \tau^{\prime}, \iota^{\prime}$ are

$$
\begin{aligned}
& \delta^{\prime}=\frac{1}{2}[M-3(\alpha-1)], \\
& \kappa^{\prime}=\quad \alpha-1, \\
& \tau^{\prime}=\frac{1}{2}[N-3(\beta-1)], \\
& \iota^{\prime}=r \quad \beta-1 .
\end{aligned}
$$


I say that a singularity is simple when we have one branch, compound when we have more than one branch; the case above considered is that of a simple singularity, viz. we have on the curve one point, one tangent, one branch.

We may have a compound singularity where the branches all touch, that is we may have one point, one tangent, several branches. It may be seen that if $\frac{1}{2} M$ denote the number of common points of all the branches (that is of each branch with itself, and of every two branches with each other), and in like manner if $\frac{1}{2} N$ denote the number of common tangents of all the branches (that is of each branch with itself, and of every two branches with each other), then the formulæ are

$$
\begin{aligned}
& \delta^{\prime}=\frac{1}{2}[M-3 \Sigma(\alpha-1)], \\
& \kappa^{\prime}=\quad \Sigma(\alpha-1), \\
& \tau^{\prime}=\frac{1}{2}[N-3 \Sigma(\beta-1)], \\
& \iota^{\prime}=\quad \Sigma(\beta-1),
\end{aligned}
$$

where the signs $\Sigma$ refer to the different branches.

Again, we may have a compound singularity, one point, several tangents with to each of them a branch or branches; here if $M$ denote the number of the common points of all the branches, and $N$ the number of the common tangents of all the branches belonging to any one tangent, then the formulæ are

$$
\begin{array}{lr}
\delta^{\prime}=\frac{1}{2} & {\left[M-3 \Sigma^{\prime} \Sigma(\alpha-1)\right],} \\
\kappa^{\prime}= & \Sigma^{\prime} \Sigma(\alpha-1), \\
\tau^{\prime}=\frac{1}{2} \Sigma^{\prime}[N-3 \Sigma(\beta-1)], \\
\iota^{\prime}= & \Sigma^{\prime} \Sigma^{\prime}(\beta-1),
\end{array}
$$

where the signs $\Sigma$ refer to all the branches belonging to the same tangent, and the signs $\Sigma^{\prime}$ to the different tangents. It is to be remarked, that the point on the curve is equivalent to the $\delta^{\prime}$ double points and $\kappa^{\prime}$ cusps; each tangent is equivalent to $\frac{1}{2}[N-3 \Sigma(\beta-1)]$ double tangents, and $\Sigma(\beta-1)$ inflexions, the numbers $N, \beta$ referring of course to the tangent in question.

Lastly, we may have a compound singularity, one tangent, several points (of contact), with to each of them a branch or branches; here if $N$ denote the number of the common tangents of all the branches, $M$ the number of the common points of all the branches belonging to any one point of contact, the formulæ are

$$
\begin{aligned}
& \delta^{\prime}=\frac{1}{2} \Sigma^{\prime}\left[\begin{array}{ll}
(M-3 \Sigma & (\alpha-1)],
\end{array}\right. \\
& \kappa^{\prime}=\quad \Sigma^{\prime} \Sigma(\alpha-1) \text {, } \\
& \tau^{\prime}=\frac{1}{2} \quad\left[\left(N-3 \Sigma \Sigma^{\prime}(\beta-1)\right],\right. \\
& \iota^{\prime}=\quad \Sigma^{\prime} \Sigma(\beta-1) \text {, }
\end{aligned}
$$

where the signs $\Sigma$ refer to all the branches belonging to the same point of contact, and the signs $\Sigma^{\prime}$ to the different points of contact; it is to be remarked that the 
tangent of the curve is equivalent to the $\tau^{\prime}$ double tangents and ' $\iota^{\prime}$ inflexions; each point of contact is equivalent to $\frac{1}{2}[M-3 \Sigma(\alpha-1)]$ double points and $\Sigma(\alpha-1)$ cusps, the numbers $M, \alpha$ referring of course to the point of contact in question.

There is no difficulty in passing to the case of the compound singularity when the formulæ for the simple singularity, one point, one tangent, one branch, are once obtained, and I now go back to the consideration of this case.

The class of a curve is equal to the number of tangents which can be drawn through an arbitrary point: the points of contact of these tangents are given as the intersections of the curve with a certain curve, the polar of the arbitrary point in regard to the curve; this polar passes through each double point and cusp, the double point counting as two points of intersection, and the cusp as three points of intersection (this is in fact the theory by which is found the reduction $=2 \delta+3 \kappa$ in the class of the curve). Hence, if the curve has a singularity $\left(\delta^{\prime}, \kappa^{\prime}, \tau^{\prime}, \iota^{\prime}\right)$, which to fix the ideas may be assumed to be a simple singularity, 'one point, one tangent, one branch '; then the polar passes through the singular point, the number of intersections being $2 \delta^{\prime}+3 \kappa^{\prime}$, or if the actual number of intersections be $M$, then we have $M=2 \delta^{\prime}+3 \kappa^{\prime}$. It is to be shown that the number $M$ is equal to twice the number of common points which the curve has with itself at the singular point, so that the last-mentioned number is $=\frac{1}{2} M$. Suppose in the first instance that there is only a single branch, and let the branch be given by the equation

$$
P=y+A x^{p}+B x^{q}+\ldots=0,
$$

or introducing for homogeneity the third coordinate $z$, let this equation be

$$
P=y z^{-1}+A x^{p} z^{-p}+B x^{q} z^{-q} \ldots=0,
$$

and let $P_{1}=0, P_{2}=0, \ldots P_{\alpha}=0$, be the corresponding equations for the component partial branches; it is allowable to write $P_{1} P_{2} \ldots P_{\alpha}=0$ for the equation of the curve $\left({ }^{1}\right)$. Hence if $(\alpha, \beta, \gamma)$ be the coordinates of the arbitrary point, or putting in the first instance $\gamma=1$, if $(\alpha, \beta, 1)$ be the coordinates, then writing $\Delta=\alpha \delta_{x}+\beta \delta_{y}+\delta_{z}$, the equation of the polar is $\Delta P_{1} P_{2} \ldots P_{a}=0$, or, what is the same thing,

and we have

$$
P_{2} P_{3} \ldots P_{a} \Delta P_{1}+P_{1} P_{3} \ldots P_{a} \Delta P_{2}+\text { \&c. }=0 \text {, }
$$

$$
\Delta P=\alpha\left(p A x^{p-1} z^{-p}+q B x^{q-1} z^{-q} \ldots\right)+\beta z^{-1}-\left(p A x^{p} z^{-p-1}+q B x^{q} z^{-q-1} \ldots\right),
$$

or putting $z=1$, this is

$$
\Delta P=\alpha\left(p A x^{p-1} \quad+q B x^{q-1} \quad \ldots\right)+\beta \quad-\left(p A x^{p} \quad+q B x^{q} \quad \ldots\right),
$$

and we have thence the values of $\Delta P_{1}, \Delta P_{2} \ldots \Delta P_{\alpha}$; the thing to be observed is, that the equation $\Delta P=0$ is not satisfied (and therefore also each of the equations $\Delta P_{1}=0, \ldots \Delta P_{a}=0$ is not satisfied) by the coordinates $x=0, y=0$ of the singular point. We have now with the equation $\Delta P_{1} P_{2} \ldots P_{a}=0$ of the polar to combine the

1 Of course this is not the equation in its rational and integral form, and on this account the reasoning of the text is not free from difficulty; the same remark applies to a subsequent equation. 
equation $P_{1} P_{2} \ldots P_{\alpha}=0$ : the last-mentioned equation breaks up into the equations $P_{1}=0, P_{2}=0, \ldots P_{a}=0$; and selecting for example the equation $P_{1}=0$, this gives the system $P_{1}=0, P_{2} P_{3} \ldots P_{a} \Delta P_{1}=0$, or since we require only the intersections at the singular point, and $\Delta P_{1}=0$ does not pass through this point, this may be replaced by $P_{1}=0, P_{2} P_{3} \ldots P_{a}=0$. The complete system is thus $\left(P_{1}=0, P_{2} P_{3} \ldots P_{\alpha}=0\right)$, $\left(P_{2}=0, P_{1} P_{3} \ldots P_{a}=0\right), \ldots\left(P_{a}=0, P_{1} P_{2} \ldots P_{a-1}=0\right)$; or, what is the same thing, we have each pair $\left(P_{r}=0, P_{s}=0\right)$ taken twice. To eliminate $y$ from these equations, we have merely to write $P_{r}-P_{s}=0$, or, what is the same thing, we have $\zeta\left(P_{1}, P_{2} \ldots P_{a}\right)=0$, $\zeta$ denoting the product of the squares of the differences of the functions $\left(P_{1}, P_{2} \ldots P_{a}\right)$. Suppose that any two partial branches $P_{r}=0, P_{s}=0$ intersect (according to the above-mentioned definition) in $p$ points; then $P_{r}-P_{s}$ contains the factor $x^{p}$, and hence the product $\zeta\left(P_{1}, P_{2} \ldots P_{a}\right)$ contains as a factor $x$ to the power $2 \Sigma p$, that is, the equation in $x$ has $2 \Sigma p$ roots each $=0$. Whence if $\Sigma p=\frac{1}{2} M$, then the equation in $x$ has $M$ roots each $=0$, or the curve and polar have at the singular point $M$ intersections, that is $M=2 \delta^{\prime}+3 \kappa^{\prime}$.

I have no complete proof to offer of the remaining equation $\kappa^{\prime}=\alpha-1$, it was obtained from the consideration of a particular case as follows. Consider the linear branch $y=A x^{p}+\ldots$, where the exponents are all positive integers, and taking the axis of $x$ to be the tangent, the least exponent $p$ is greater than unity; if $p=2$ there is at the origin no inflexion, if $p=3$ there is a single inflexion, and generally the number of inflexions is $=p-2$. Now it will presently appear that in line-coordinates the equation of the branch is $Z=A^{\prime} X^{\frac{p}{p-1}}$, or replacing $Z, X$ by the original point-coordinates $y, x$ the branch $y=A^{\prime} x^{\frac{p}{p-1}}+\ldots$ has at the origin $p-2$ cusps; but in the branch in question we have $\alpha=p-1$, and the number of cusps is thus $=\alpha-1$; this result is confirmed by other particular instances, and I assume in general that we have $\kappa^{\prime}=\alpha-1$; whence in the case of a simple singularity, or where there is only one branch we have $M=2 \delta^{\prime}+3 \kappa^{\prime}, \kappa^{\prime}=\alpha-1$, or, what is the same thing, $\delta^{\prime}=\frac{1}{2}[M-3(\alpha-1)], \kappa^{\prime}=\alpha-1$. The reasoning is easily adapted to the case of a compound singularity.

I consider the branch

$$
y+A x^{p}+B x^{q}+\ldots=0,
$$

(where it is assumed that the axis of $x$ is a tangent to the branch, and therefore that the lowest exponent $p$ is greater than unity), introducing the coordinate $z$ for homogeneity, this becomes

$$
y z^{-1}+A x^{p} z^{-p}+B x^{q} z^{-q}+\ldots=0,
$$

and I proceed to find the corresponding equation in line coordinates, taking these to be $X, Y, Z$, we have

$$
\begin{aligned}
& \lambda X=p A x^{p-1} z^{-p}+B q^{x^{-1}} z^{-q}+\ldots, \\
& \lambda Y=\quad z^{-1}, \\
& \lambda Z=-\quad y z^{-2}-p A x^{p} z^{-p-1}-q B x^{q} z^{-q-1}+\ldots
\end{aligned}
$$


or writing $z=1, Y=1$, we find $\lambda=1$, and therefore

$$
\begin{array}{ll}
X= & p A x^{p-1}+ \\
Z B x^{q-1}+\ldots \\
Z=-y-p A x^{p}- & q B x^{q}+\ldots
\end{array}
$$

here substituting for $-y$ its value $=A x^{p}+B x^{q}+\ldots$, we have

$$
\begin{aligned}
& X=\quad p A x^{p-1}+\quad q B x^{q-1}+\ldots \\
& Z=(1-p) A x^{p}+(1-q) B x^{q}+\ldots
\end{aligned}
$$

Hence writing $p A x^{p-1}=\theta$, the equations are

$$
\begin{aligned}
& X=\theta-B^{\prime \prime} \theta^{q-1}-\ldots \\
& Z=-A^{\prime} \theta^{-p-1}-B^{\prime} \theta^{\frac{q}{p-1}}-\ldots
\end{aligned}
$$

so that eliminating $\theta$, we have

$$
Z=A^{\prime} X^{\frac{p}{p-1}}+B^{\prime} X^{\frac{q}{p-1}}+\ldots
$$

and it is easy to see by Lagrange's theorem, that the general form of the exponents in the series on the right-hand side is $\frac{p+f(q-p)+g(r-p)+\ldots}{p-1}$, where $f, g, \ldots$ are positive integers, zero included. The equation in line-coordinates being known, the subsequent investigation is precisely the same as that for the point-coordinates, and hence in the case of one branch, if this be in regard to its tangents $\beta$-ic, and have $\frac{1}{2} N$ common tangents with itself, then $2 \tau^{\prime}+3 \iota^{\prime}=N, \iota^{\prime}=\beta-1$, or, what is the same thing, $\tau^{\prime}=\frac{1}{2}[N-3(\beta-1)], \iota^{\prime}=\beta-1$. The investigation in the case of a simple singularity of the values of $\delta^{\prime}, \kappa^{\prime}, \tau^{\prime}, \iota^{\prime}$ is thus completed. 